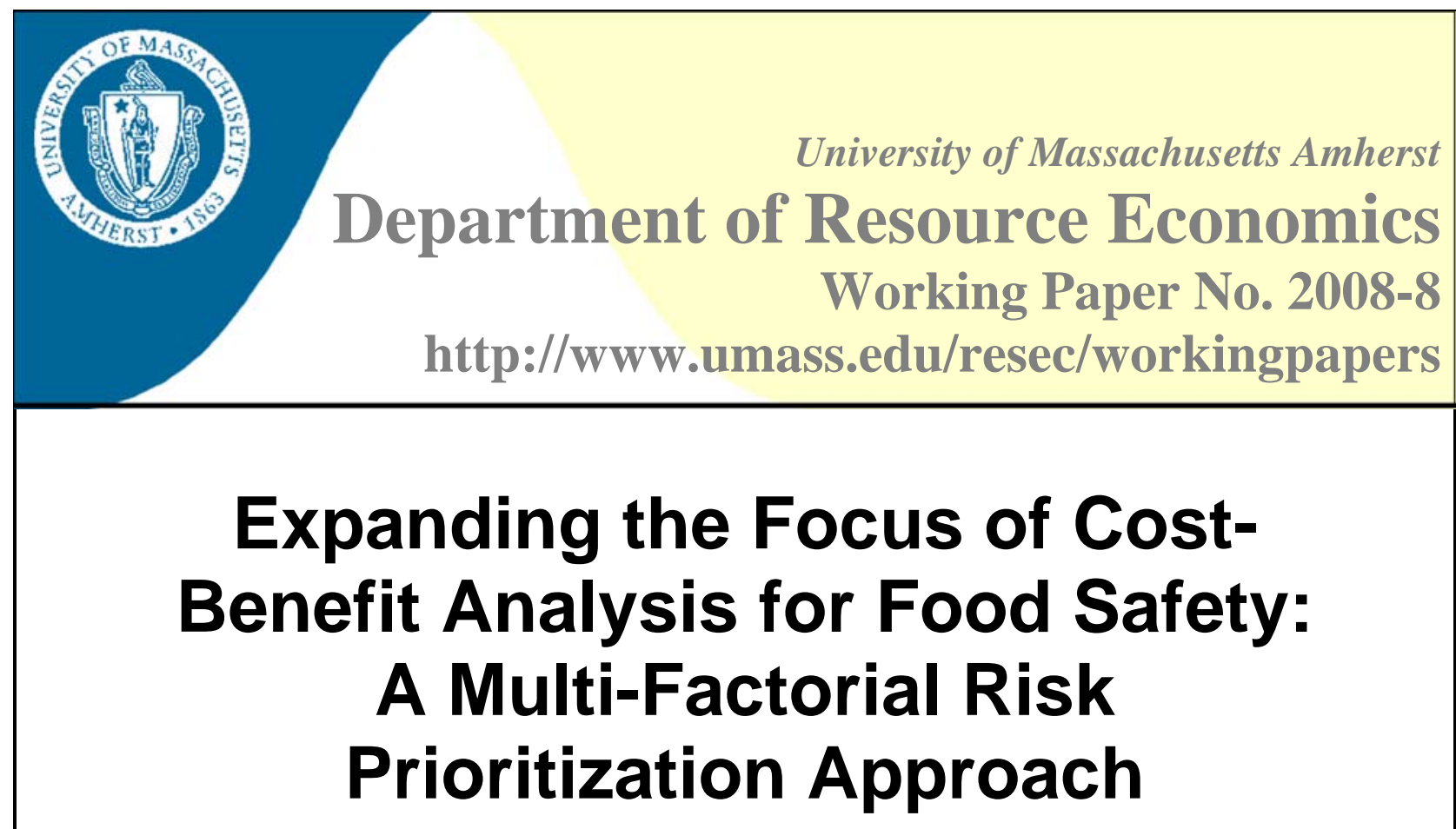

Julie A. Caswell ${ }^{1}$

Abstract:

A pressing need in the area of food safety is a tool for making overall, macro judgments about which risks should be given priority for management. Governments often seek to base this prioritization on public health impacts only to find that other considerations also influence the prioritization process. A multi-factorial approach formally recognizes that public health, market-level impacts, consumer risk preferences and acceptance, and the social sensitivity of particular risks all play a role in prioritization. It also provides decision makers with a variety of information outputs that allow risk prioritization to be considered along different dimensions. Macro-level prioritization of risks based on multiple factors is an important expanded use of cost-benefit analysis to manage risk.

Keywords: cost-benefit analysis, food safety, risk prioritization

JEL Classification: I18, L51, Q18, K32, H11

${ }^{1}$ Julie A. Caswell, Department of Resource Economics

University of Massachusetts, 215 Stockbridge Hall

80 Campus Center Way, Amherst, MA 01003-9246

E: caswell@resecon.umass.edu P: 413-545-5735 F: 413-545-5853 


\title{
Expanding the Focus of Cost-Benefit Analysis for Food Safety: A Multi-Factorial Risk Prioritization Approach
}

\author{
Julie A. Caswell \\ Department of Resource Economics \\ University of Massachusetts Amherst \\ 80 Campus Center Way \\ 215 Stockbridge Hall \\ Amherst, MA 01003 \\ Phone: (413) 545-5735 \\ Fax: (413) 545-5853 \\ E-mail: caswell@resecon.umass.edu
}

Acknowledgement: This paper is based on joint work with several colleagues. Please see: Henson, Spencer J., Julie A. Caswell, John A. L. Cranfield, Aamir Frazil, Valerie J. Davidson, Sven M. Anders, and Claudia Schmidt. 2007. A Multi-Factorial Risk Prioritization Framework for Food-Borne Pathogens. Working Paper 2007-8. Department of Resource Economics, University of Massachusetts Amherst. http://people.umass.edu/resec/workingpapers/documents/ResEcWorkingPaper2007-8.pdf 


\title{
Expanding the Focus of Cost-Benefit Analysis for Food Safety: A Multi-Factorial Risk Prioritization Approach
}

\begin{abstract}
:
A pressing need in the area of food safety is a tool for making overall, macro judgments about which risks should be given priority for management. Governments often seek to base this prioritization on public health impacts only to find that other considerations also influence the prioritization process. A multi-factorial approach formally recognizes that public health, marketlevel impacts, consumer risk preferences and acceptance, and the social sensitivity of particular risks all play a role in prioritization. It also provides decision makers with a variety of information outputs that allow risk prioritization to be considered along different dimensions. Macro-level prioritization of risks based on multiple factors is an important expanded use of cost-benefit analysis to manage risk.
\end{abstract}

Keywords: cost-benefit analysis, food safety, risk prioritization 


\section{Expanding the Focus of Cost-Benefit Analysis for Food Safety: A Multi-Factorial Risk Prioritization Approach}

\section{Background}

Governments around the world give support to the idea that risk management for food safety should be science based. A great deal of effort has gone into defining what science-based management means in principle and in practice (e.g., Health Canada 2000, NZFSA 2000, FAO/WHO 2002, Batz et al. 2004). A prominent example of the implementation of sciencebased principles is the Agreement on the Application of Sanitary and Phytosanitary Measures (SPS Agreement) under the World Trade Organization (WTO), which gives a central role to risk assessment.

A major hurdle in making science-based regulatory choices is that political leaders and risk managers who work in government must respond to a broad range of factors in making decisions. The most prominent of these is public health. However, one would be hard pressed to explain the decisions of regulatory agencies based solely on the management of rates of illness and death. Other factors are also important, including the impact of a risk and its management on industry, the concern consumers express about a risk, and sensitivity about who is impacted by the risk and how. Limited resources mean that only a subset of food safety risks can be given priority in management plans.

It may be manageable to outline what science -based decision making looks like when the focus in making decisions is only on the public health aspects of food safety. But what might it look like if the approach is expanded to consider the other important factors that affect risk management choices? Such an approach would make the factors affecting decisions more explicit and the process more transparent and defensible. 


\section{An Expanded Approach to Prioritizing Risks}

A first-stage version of an expanded approach to food safety risk management has been developed by a group of researchers at the Public Health Agency of Canada, the University of Guelph, and the University of Massachusetts Amherst (Henson, Caswell, Cranfield, Frazil, Davidson, Anders, and Schmidt 2007). The discussion here is based on this Multi-Factorial Risk Prioritization Framework and the work of this group that attempts to incorporate all dimensions of the decision-making environment into a single decision tool.

A pressing need in the area of food safety is a tool for making overall, macro judgments about which risks should be given priority for management. It is important to the degree possible that such a tool incorporate all the considerations that ultimately influence decisions. What happens when this is not the case? A frequent scenario has governments and their regulatory agencies building systems to base priorities on public health concerns only to find that the priorities decided upon do not correspond as closely as they might have anticipated to those dictated by such health concerns. Why have the system if it is not used? The answer is likely to be that it is being used but in conjunction with an additional set of factors that modify the public health priorities. Adding even further confusion is the likelihood the priorities have been reordered based on industry or micro factors. An example is ad hoc consideration of assessments of the feasibility of risk management options. A risk that is lower on the list in terms of public health impact might move up in the priorities because it is perceived that an effective risk management approach already exists. The exclusion of important factors that influence decisions and the mixing of macro- and micro-level information may lead to incoherent decisions or at least to decisions that appear to be incoherent. 


\section{Orientation of the Framework}

An expanded approach to decision making, and ultimately to cost-benefit analysis, such as the Multi-Factorial Risk Prioritization Framework incorporates all dimensions of the decisionmaking environment into the decision tool. It also provides decision makers with a variety of information outputs that allow risk prioritization to be considered along different dimensions. The latter is important for at least two reasons. First, different ways of presenting information allow decision makers to appreciate the different dimensions of risks. Second, information to make decisions is always incomplete. Presenting a single "result" of the prioritization process tends to imply much more certainty than actually exists.

How does an expanded approach to risk prioritization such as proposed by the MultiFactorial Risk Prioritization Framework fit with the call for science-based regulatory decision making? My view is that it extends the scientific base for decision making beyond the confines of risk assessment into the development of a broad set of information useful in setting priorities. This set of information is contributed by the life and social sciences. The goal is more coherent decision making.

\section{Focusing on Food-Pathogen Pairings and Multiple Factors}

The Multi-Factorial Risk Prioritization Framework uses food-pathogen pairings (e.g., beef and E. coli O157:H7) as its basic unit of analysis, as do many other approaches to prioritization and to attribution of food safety related illness and deaths. An advantage of using this basic unit of analysis is that information can subsequently be aggregated by food for all pathogens or by pathogen for all foods. A disadvantage is that many cells in the food-pathogen matrix will be lacking in information. However, using this breakdown makes it clear where important information is missing. 
An expanded approach to risk prioritization needs to capture all the relevant factors that ultimately influence risk management decisions. Clearly there are many ways that one might try to capture these factors. The Multi-Factorial Risk Prioritization Framework does so by first including the central criterion of public health impacts, measured in the recognized approaches of Disability Adjusted Life Years (DALYs) and cost of illness. A second factor focuses on consumer risk perceptions and acceptance to capture differences in priority setting that may be responsive to differences in consumers' views of the seriousness of particular risks and their acceptance of them. A third factor is the market level impacts of a risk and its management. For example, the size of an industry domestically, how much it exports, or the number of people it employs may affect priorities for managing risks it poses. Finally, a fourth factor is social sensitivity. This factor captures risks that societies place more importance on managing, for example, risk that affect pregnant women or children.

This set of factors, or another set that tries to reflect the same range, clearly expands the analytical focus of risk prioritization and cost-benefit analysis for food safety. Objections to this approach may be that it increases the information burden and raises questions about how to aggregate across factors to get 'an answer'. However, since this range of factors is already being considered by risk managers, albeit usually on an ad hoc basis, the scope of information to be used has not really expanded but the quality of the information would need to be improved. The need to aggregate across factors is not an issue if it is recognized early on that the factors are indeed multi-dimensional and cannot be aggregated.

\section{Presenting Information for Prioritization}

Organizing and presenting relevant data is a key challenge for efforts to prioritize food safety risks for management purposes. As noted above, the Multi-Factorial Risk Prioritization 
Framework visualizes food-pathogen pairings as the basic unit of analysis and within each pairing it proposes a separate information card showing food-pathogen-factor information. These information cards can be used directly by decision makers to understand the underlying data.

Information on the multiple factors should be presented in additional ways to decision makers. A simple but effective way is to use cobweb drawings to show how different foodpathogen combinations compare across factors. Figure 1 shows an example where the rays from the center of the cobweb represent four separate factors. An alternative, more formal analytical approach is to use multiple criteria decision analysis to set criteria and rank risks based on those criteria. Experience will indicate which set of formats is most helpful to decision makers. In general, different formats are likely to be complementary to each other rather than being substitutes in the delivery of information.

\section{Figure 1. A Graphical Approach to Presenting Risk Profiles}

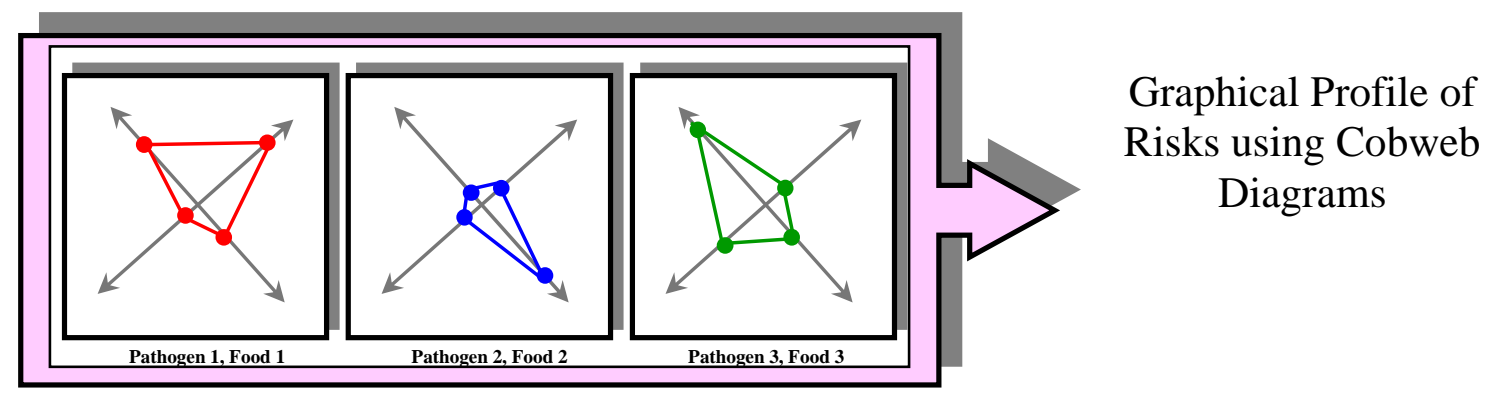

Source: Henson, Caswell, Cranfield, Frazil, Davidson, Anders, and Schmidt 2007, p. 34.

\section{Developing Outputs from the Prioritization Process}

Risk prioritization is by its nature an iterative process; new information calls for the systematic updating of the analysis. A first output envisioned from use of an approach such as the Multi-Factorial Risk Prioritization Framework by risk managers is a list of food-pathogen 
risks based on criteria that they apply. Work by Henson et al. (2007) suggests that this list be made without considering either feasibility analysis for possible management strategies or uncertainty analysis. Feasibility and uncertainty analysis can then be systematically applied as a second stage to generate lists that take these factors into consideration.

\section{Conclusions}

Cost-benefit analysis can be used at many levels to evaluate food safety regulations. It is most often thought about and done on a micro level. For example, what are the costs and benefits of two alternative interventions for controlling the health risks associated with a particular foodpathogen combination? More generally, however, at the macro-level prioritization of risks for management purposes in the area of food safety is a key form of cost-benefit analysis. Good macro level analysis is essential to identify the specific food-pathogen combinations and intervention strategies that will receive the micro-level cost-benefit analysis.

Good cost-benefit analysis at the micro level has long made a concerted effort to measure all the life and social science factors that influence the ultimate impact of a food safety regulation. Expanding the scope of cost-benefit analysis is also important at the macro level of analysis. At this level, risk managers do in fact recognize multiple factors in setting priorities. Public health is key but factors such as consumer risk perceptions and acceptance, market level impacts, and social sensitivity are also influential. However, these additional factors are often brought in on an ad hoc basis, which undermines the reliability of the analysis. The quality of macro-level decision making on priorities for food safety regulation can be aided by a comprehensive framework that explicitly considers the multiple factors that influence the benefits and costs of risk management. 


\section{References}

Batz, M.B., S. Hoffmann, A.J. Krupnick, J.G. Morris, D.M. Sherman, M.R. Taylor and J.S. Tick (2004) Identifying the Most Significant Microbiological Food-Borne Hazards to Public Health: A New Risk Ranking Model. Food Safety Research Consortium Discussion Paper Series No. 1.

FAO/WHO (2002) Principles and Guidelines for Incorporating Microbiological Risk Assessment in the Development of Food Safety Standards and Related Texts. Report, Joint FAO/WHO consultations Kiel, 18. - 22. March 2002, Kiel, Germany.

Health Canada (2000) Health Canada Decision-making Framework for Identifying, Assessing and Managing Health Risks. Health Canada, Ottawa.

Henson, Spencer J., Julie A. Caswell, John A. L. Cranfield, Aamir Frazil, Valerie J. Davidson, Sven M. Anders, and Claudia Schmidt. 2007. A Multi-Factorial Risk Prioritization Framework for Food-Borne Pathogens. Working Paper 2007-8. Department of Resource Economics, University of Massachusetts Amherst. Accessed June 1, 2007. http://people.umass.edu/resec/workingpapers/documents/ResEcWorkingPaper2007-8.pdf NZFSA (2000) Food Administration in New Zealand - A Risk Management Framework for Food Safety. Joint Ministry of Health and Ministry of Agriculture and Forestry Food Harmonization Project. Available at: http://www.moh.govt.nz. Accessed September 15, 2006. 